\title{
Effect of Sitagliptin and Glimepiride on Glucose Homeostasis and cAMP Levels in Peripheral Tissues of HFD/STZ Diabetic Rats
}

\author{
Mohamed I Saad $^{1,{ }^{*}}$, Maher A Kamel ${ }^{1}$, Mervat Y Hanafi ${ }^{1}$, Madiha H Helmy ${ }^{1}$, Rowaida R Shehata ${ }^{2}$ \\ ${ }^{1}$ Department of Biochemistry, Medical Research Institute, Alexandria University, Egypt \\ ${ }^{2}$ Department of Pharmacology, Medical Research Institute, Alexandria University, Egypt \\ *Corresponding author: m.ibrahim1988@hotmail.com
}

Received August 20, 2014; Revised September 02, 2014; Accepted September 11, 2014

\begin{abstract}
Introduction: T2DM is a group of metabolic disorders manifested by hyperglycemia as a result of insulin insufficiency and/or resistance. The main goal of antidiabetic therapies is to lower glucose levels, and therefore prevent development of diabetes complications. DPP-4 inhibitors (e.g. sitagliptin) are relatively new antidiabetic drugs which inhibit the activity of DPP-4 enzyme and therefore prevent rapid degradation of incretin hormones. Objective: We investigated effects of sitagliptin on glucose homeostasis, lipid profile, and insulin signaling by determination of cAMP levels in peripheral tissues of HFD/STZ diabetic rats, compared to glimepiride. Methods: The experimental rats were divided into five groups, each group comprising 10 rats. Group (1) served as the normal control rats and administered DMSO (without treatments) as the vehicle. The rest of the groups were rendered diabetic by feeding HFD containing $40 \%$ fats for 4 weeks, followed by a single I.P. injection of STZ (45 $\mathrm{mg} / \mathrm{kg}$ of body weight). One week after STZ injection, the rats with FBG level of $\geq 200 \mathrm{mg} / \mathrm{dl}$ were considered diabetic. Group (2) served as the diabetic untreated rats and administered DMSO (without treatments) as the vehicle. Group (3) served as diabetic rats treated with glimepiride ( $0.1 \mathrm{mg} / \mathrm{kg}$ of body weight). Group (4) and group (5) served as diabetic rats treated with sitagliptin (10 and $30 \mathrm{mg} / \mathrm{kg}$ of body weight, respectively). Treatments were dissolved in DMSO and were given orally for 4 weeks. At the end of the treatment period, the blood, liver and adipose tissues (White and brown) were collected for biochemical analysis. Results: In normal control rats, the highest content of cAMP was observed in BAT. Diabetic rats showed an elevation in cAMP levels of liver and WAT to be 1.3 and 3.9 fold control values, respectively, while in BAT, cAMP level decreased to be 0.4 fold control value. Sitagliptin and glimepiride significantly decreased cAMP levels in liver and WAT. Conclusion: We conclude that sitagliptin and glimepiride have comparable effects on glucose homeostasis. Both drugs have cAMP-lowering effect which may suggest their potential protecting effect against vascular complications of diabetes.
\end{abstract}

Keywords: diabetes, DPP-4 inhibitors, sitagliptin, glimepiride, cAMP

Cite This Article: Mohamed I Saad, Maher A Kamel, Mervat Y Hanafi, Madiha H Helmy, and Rowaida R Shehata, "Effect of Sitagliptin and Glimepiride on Glucose Homeostasis and cAMP Levels in Peripheral Tissues of HFD/STZ Diabetic Rats.” American Journal of Biomedical Research, vol. 2, no. 3 (2014): 52-60. doi: 10.12691/ajbr-2-3-3.

\section{Introduction}

Type 2 diabetes mellitus (T2DM) is a group of metabolic disorders manifested by hyperglycemia as a result of insulin insufficiency and/or insulin resistance. Insulin resistance is the inability of insulin to exert its action on cells. The pathophysiology of T2DM is not fully understood and there are theories link T2DM with insulin signaling defects, level of reactive oxygen species and obesity. T2DM could result in deleterious complications such as cardiovascular disorders, renal failure, retinopathy and poor wound healing [1].

Insulin is the main anabolic hormone which secreted from beta $(\beta)$-cells of the pancreas. It is released in response to increased nutrients level in blood e.g. glucose and amino acids [2]. It regulates the whole body fuel homeostasis through its action on liver, skeletal muscles and adipose tissues. It stimulates glucose uptake, glycogenesis, fatty acid biosynthesis and adipogenesis, whereas it inhibits glycogenolysis, gluconeogenesis and lipolysis. Insulin exerts its actions through a matrix of interacting pathways, allowing for extensive modulation and divergence in signal transduction, which starts with insulin binding to its cell membrane tyrosine kinase receptors [3]. Important components in insulin signaling are insulin receptors, insulin receptor substrate proteins (IRS), phosphatidylinositol-3 ( $\left.\mathrm{PI}_{3}\right)$ kinase, Akt/protein kinase $\mathrm{B}$ (Akt/PKB), and mitogen activated protein kinase (MAPK) [3]. $\mathrm{PI}_{3}$ kinase is the critical component of insulin signaling, mediating the metabolic effect of insulin through activation of Akt/PKB and atypical protein kinase C (aPKC) pathways [4]. 
Adipose tissues play an important role in the development of insulin resistance and T2DM. There are two kinds of adipose tissue: white adipose tissue (WAT) and brown adipose tissue (BAT). WAT is the primary site of energy storage in the form of triglycerides, whereas BAT contains multilocular adipocytes which contains a large number of mitochondria and plays a substantial role in non-shivering thermogenesis [5]. Sympathetic nerve endings release noradrenaline in the proximity of brown fat cells, where noradrenaline activates G-protein-coupled $\beta$-adrenergic receptors initiating a cascade of metabolic events resulting in the activation of uncoupling protein 1 (UCP1) [6]. Insulin antagonizes hormone-induced lipolysis - caused by counter-regulatory hormones e.g. glucagon mainly by activation of phosphodiesterase 3B (PDE3B) via Akt/PKB that degrades intracellular cAMP [3].

Dipeptidyl peptidase-4 inhibitors are relatively new antidiabetic drugs which inhibit the enzymatic activity of dipeptidyl peptidase-4 (DPP-4) that is responsible for rapid degradation of the incretin hormones: glucosedependent insulinotropic peptide (GIP) and glucagon-like peptide-1 (GLP-1) [7]. DPP-4 inhibitors, therefore, causes stimulation of insulin secretion, inhibition of glucagon secretion and preservation of $\beta$-cell mass through stimulation of cell proliferation and inhibition of apoptosis [8]. Sitagliptin, The first DPP-4 inhibitor to be approved in the USA for the treatment of T2DM, is used nowadays at a dose of $100 \mathrm{mg} /$ day as a monotherapy or in combination with other drugs [7]. Although, the effect of sitagliptin on insulin secretion is well-defined; its effect on insulin action in the peripheral tissues is not elucidated.

The objective of the present study is to investigate the effect of sitagliptin on glucose homeostasis parameters (e.g. blood glucose level, serum insulin, insulin resistance using the homeostasis model assessment), lipid profile, and insulin signaling by determination of cAMP levels in liver and adipose tissues. This study was performed on high-fat diet (HFD)/streptozotocin (STZ)-induced diabetic rats in which the effect of sitagliptin was compared to that of the antidiabetic sulfonylurea (glimepiride).

\section{Materials and Methods}

\subsection{Experimental Animals}

Wistar rats aged 3 - 4 months were purchased from the Medical Technology Center (Alexandria, Egypt). The rats were housed at a temperature of $23 \pm 1^{\circ} \mathrm{C}$ with $12 / 12 \mathrm{~h}$ light/dark cycles and $45 \pm 5 \%$ humidity with free access to water and chow diet for a week prior to the experiment.

\subsection{Experimental Design}

The experimental animals were divided into five groups, each group comprising 10 rats detailed as follows: Group (1) served as the normal control rats and administered dimethyl sulfoxide (DMSO) as a vehicle without any treatments. The rest of animals were rendered diabetic by feeding a high-fat diet containing 40\% fats (HFD) for 4 weeks, followed by a single intraperitoneal injection of STZ at a low dose (45 mg/kg of body weight, dissolved in $0.05 \mathrm{M}$ citrate buffer, $\mathrm{pH} 4.5$, immediately before use). One week after injection, fasting blood glucose (FBG) levels were determined from tail blood using an AccuCheck Active glucometer (Roche Diagnostics, Manheim, Germany). The rats with FBG levels above $200 \mathrm{mg} / \mathrm{dl}$ were considered as diabetic (Modified Srinivasan, et al. method) [9]. Group (2) served as the diabetic untreated rats and administered (DMSO) as a vehicle without any treatments. Group (3) served as diabetic rats treated with glimepiride ( $0.1 \mathrm{mg} / \mathrm{kg}$ of body weight). Group (4) and group (5) served as diabetic rats treated with sitagliptin (10 and $30 \mathrm{mg} / \mathrm{kg}$ of body weight, respectively). Treatments were administered daily in DMSO suspension by oral gavage for 4 weeks. The dosage was adjusted every week, according to any change in body weight to maintain similar dose per kg body weight of rat over the entire period of study for each group. FBG level was measured every week. At the end of the treatment period, the rats were fasted overnight, anaesthetized with diethyl ether and killed by cervical decapitation. The blood was collected for serum separation and biochemical analysis. Liver and adipose tissues (WAT and BAT) were excised immediately and stored at $-80^{\circ} \mathrm{C}$. WAT was obtained from visceral (retroperitoneal) adipose depots, while BAT was obtained from adipose depots in the interscapular region.

\subsection{Oral Glucose Tolerance Test (OGTT)}

The OGTT was performed at the end of treatment period (4 weeks). After overnight fasting for 12 hours, the animals were administered glucose $(2.5 \mathrm{~g} / \mathrm{kg}$ of body weight) dissolved in water by oral gavage. Blood glucose levels were determined from the tail vein with an Accucheck Active glucometer at 0, 30, 60, 90, and 120 minutes.

\subsection{Determination of Glucose, Insulin and Lipid Profile}

The level of serum glucose was estimated using an Accu-Check Active glucometer. Serum insulin level was assayed using a commercial kit (Millipore ${ }^{\circledR}$ ) according to the manufacturer's instructions. This assay is a sandwich ELISA based on capture of insulin molecules from samples to the wells of a microtitre plate coated by pretitered amount of a monoclonal mouse anti-rat insulin antibodies and the binding of biotinylated polyclonal antibodies to captured insulin, binding of horseradish peroxidase (HRP) to the immobilized biotinylated antibodies, and quantification of the immobilized antibody-enzyme conjugates by monitoring horseradish peroxidase activities in the presence of 3,3',5,5'tetramethylbenzidine. The enzyme activity is measured spectrophotometrically by the increased absorbance at 450 nm.

Lipid profile was assessed by using a commercial diagnostic kit (Randox $(\mathrm{UK})^{\circledR}$ ) according to the manufacturer's instructions. This kit uses enzymatic methods to determine serum levels of triglycerides, total cholesterol, HDL-cholesterol. The absorbance of the standards and samples were measured spectrophotometrically at $546 \mathrm{~nm}$. LDL-cholesterol level was then calculated from the following equation:

$$
-(\text { HDL conc })-\frac{T G \text { conc }}{5}
$$$$
L D L \text { conc }=\text { Total cholesterol conc }
$$ 


\subsection{Determination of Homeostasis Model of Insulin Resistance (HOMA-IR)}

The insulin resistance index (IRI) was evaluated by homeostasis model assessment estimate of insulin resistance (HOMA-IR) as follows:

$$
I R I=\frac{\text { Fastinginsulin }(\mu I U / \mathrm{ml}) \times \text { Fastingglucose }(\mathrm{mmol} / \mathrm{L})}{22.5}
$$

\subsection{Assay of cAMP Level}

The levels of cAMP in the liver, WAT and BAT of control and experimental groups of rats were determined by $\mathrm{DRG}^{\circledR}$ Cyclic AMP direct ELISA kit according to the manufacturer's instructions. Tissue samples (liver, WAT and BAT) were frozen in liquid nitrogen and stored at $80^{\circ} \mathrm{C}$. Frozen tissues were grinded in a stainless steel mortar under liquid nitrogen until it was a fine powder. The liquid nitrogen was allowed to evaporate and the powdered tissue was weighed. $1 \mathrm{ml}$ of Sample Diluent was added for every $100 \mathrm{mg}$ of tissue and incubated for 10 minutes on ice, and then centrifuged at $\geq 600 \mathrm{x} \mathrm{g}$ at $4^{\circ} \mathrm{C}$ for 15 minutes. The supernatant was collected and the assay was done immediately. A clear microtiter plate coated with an antibody to capture sheep IgG was provided and a neutralizing plate primer solution was added to all the used wells. Standards or diluted samples were pipetted into the primed wells. A cAMP-peroxidase conjugate was added to the standards and samples in the wells. The binding reaction was initiated by the addition of a sheep antibody to cAMP to each well. After 2-hour incubation, the plate was washed and substrate was added. After a short incubation, the reaction was stopped and the intensity of the generated color was detected in a microtiter plate reader at $450 \mathrm{~nm}$. Standard plot was constructed by using standard cAMP, and then the concentrations of unknown samples were calculated from the standard curve (Figure 1). cAMP level was finally calculated in terms of protein content in each tissue sample measured by the modified Lowery, et al. method for total protein determination [10].

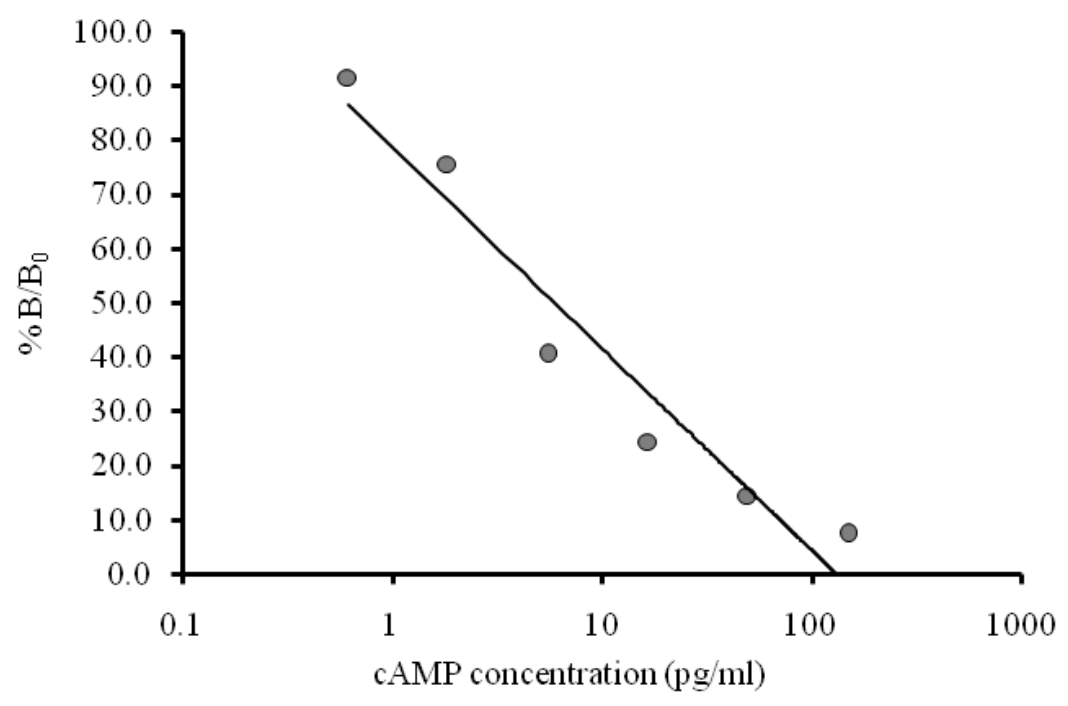

Figure 1. Standard curve of cAMP. The abbreviations denote $\mathrm{B} / \mathrm{B}_{0}$ : sample or standard bound / maximum bound

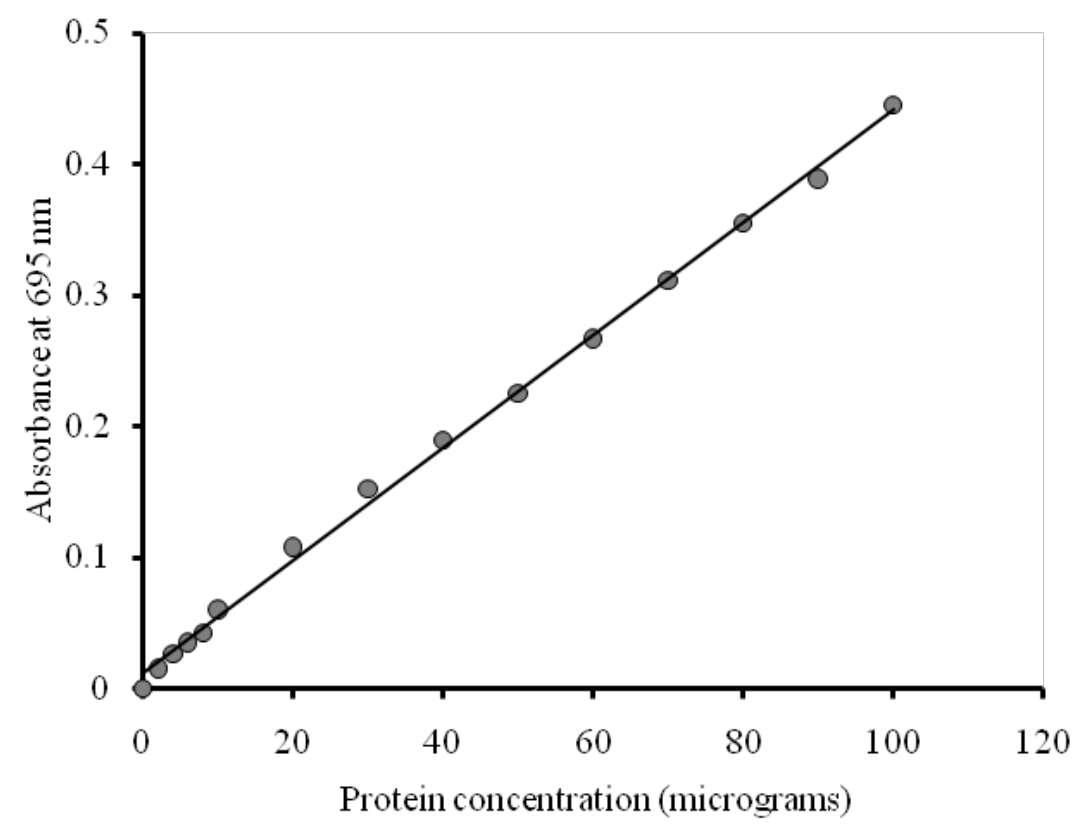

Figure 2. Standard curve of total protein 


\subsection{Determination of Total Protein Concentration}

A modification of the method of Lowery, et al. was used for the determination of protein in the samples. The color produced is thought to be due to a complex between the alkaline copper-phenol reagent and tyrosine and tryptophan residues of the protein in the sample. The absorbance was read at $695 \mathrm{~nm}$ using spectronic 21 spectrophotometer. [10] The protein concentration in each sample was estimated by referring to a standard curve (Figure 2) which was constructed using bovine serum albumin.

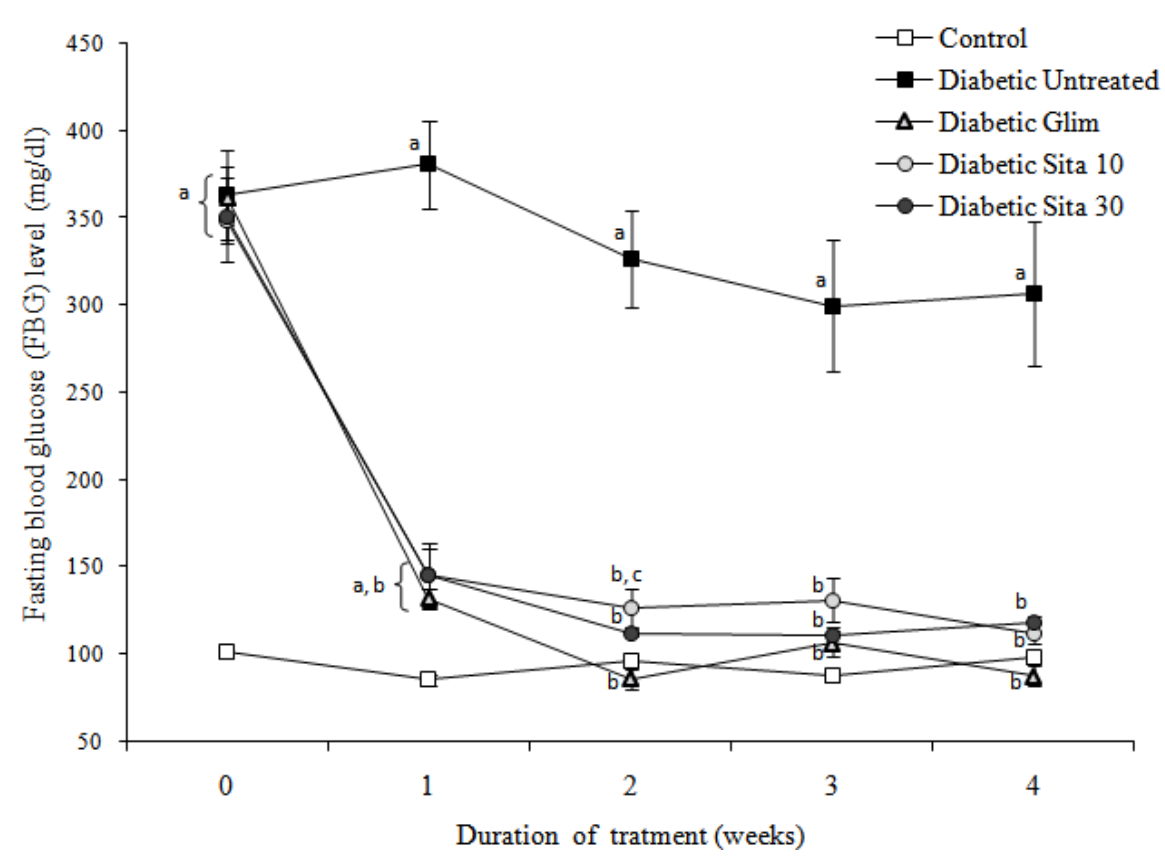

Figure 3. Change in fasting blood glucose (FBG) levels of studied groups during the treatment period (4 weeks). Values are presented as mean \pm SE (n = 10). The abbreviations denote Glim: rats treated with glimepiride $(0.1 \mathrm{mg} / \mathrm{kg})$, Sita 10: rats treated with sitagliptin (10 mg/kg) and Sita $30:$ rats treated with sitagliptin (30 mg/kg). a: significantly different from the control group, b: significantly different from the diabetic untreated group, c: significantly different from the Glim group, using ANOVA (LSD), p value $<0.05$

At the beginning of the study, all diabetic groups - after induction of diabetes and before the administration of treatments - showed significantly higher levels of FBG (hyperglycemia) compared to the control group (Table 1). Most of treated groups showed a time-dependent decrease

\subsection{Statistical Analysis}

The data were analyzed using the one-way analysis of variance (ANOVA) followed by LSD test to compare different groups with each other (SPSS software). Results were expressed as mean \pm standard error (SE) and values of $p>0.05$ were considered non-significantly different, while those of $\mathrm{p}<0.05$ were considered significant.

\section{Results}

\subsection{Glucose Homeostasis Parameters}

in FBG during the treatment period. During this time period, all of treated groups had significantly lower FBG than the untreated rats. By the end of the treatment period (after 4 weeks), glimepiride and sitagliptin at 10 and 30 $\mathrm{mg} / \mathrm{kg}$ showed nearly normal FBG levels (Figure 3).

Table 1. Baseline values of glucose homeostasis parameters and lipid profile of different studied groups

\begin{tabular}{|c|c|c|c|c|c|}
\hline \multirow{2}{*}{ Parameter } & \multirow{2}{*}{ Control } & \multicolumn{4}{|c|}{ Diabetic } \\
\hline & & Untreated & Glim & Sita 10 & Sita 30 \\
\hline Fasting blood Glucose (FBG) level (mg/dl) & $102.9 \pm 2.97$ & $\begin{array}{c}363.1 \pm 16.74 \\
\mathrm{a}\end{array}$ & $\begin{array}{c}361.9 \pm 26.76 \\
\mathrm{a}\end{array}$ & $\begin{array}{c}349.3 \pm 19.46 \\
\mathrm{a}\end{array}$ & $\begin{array}{c}362.9 \pm 16.64 \\
\mathrm{a}\end{array}$ \\
\hline Serum Insulin $(\mu \mathrm{IU} / \mathrm{ml})$ & $1.64 \pm 0.11$ & $\begin{array}{c}11.88 \pm 0.21 \\
\mathrm{a}\end{array}$ & $\begin{array}{c}11.80 \pm 0.23 \\
\mathrm{a}\end{array}$ & $\begin{array}{c}11.92 \pm 0.22 \\
a\end{array}$ & $\begin{array}{c}12.02 \pm 0.26 \\
\mathrm{a}\end{array}$ \\
\hline HOMA-IR & $0.4 \pm 0.07$ & $\begin{array}{c}10.6 \pm 0.51 \\
\mathrm{a}\end{array}$ & $\begin{array}{c}10.7 \pm 0.84 \\
\mathrm{a}\end{array}$ & $\begin{array}{c}10.2 \pm 0.49 \\
\mathrm{a}\end{array}$ & $\begin{array}{c}10.7 \pm 0.39 \\
\mathrm{a}\end{array}$ \\
\hline Triglycerides (mg/dl) & $39.5 \pm 0.45$ & $\begin{array}{c}117.8 \pm 5.4 \\
\mathrm{a}\end{array}$ & $\begin{array}{c}116.7 \pm 5.27 \\
\mathrm{a}\end{array}$ & $\begin{array}{c}118.3 \pm 5.52 \\
\mathrm{a}\end{array}$ & $\begin{array}{c}114.3 \pm 1.25 \\
\mathrm{a}\end{array}$ \\
\hline Total Cholesterol (mg/dl) & $141.8 \pm 3.02$ & $\begin{array}{c}184.9 \pm 3.04 \\
\mathrm{a}\end{array}$ & $\begin{array}{c}184.1 \pm 3.08 \\
\mathrm{a}\end{array}$ & $\begin{array}{c}183.7 \pm 3.04 \\
\mathrm{a}\end{array}$ & $\begin{array}{c}183.4 \pm 2.75 \\
\mathrm{a}\end{array}$ \\
\hline LDL-Cholesterol (LDL-C) (mg/dl) & $90.33 \pm 3.33$ & $\begin{array}{c}122.5 \pm 3.57 \\
\mathrm{a}\end{array}$ & $\begin{array}{c}121.96 \pm 4.09 \\
\mathrm{a}\end{array}$ & $\begin{array}{c}121.5 \pm 3.5 \\
\mathrm{a}\end{array}$ & $\begin{array}{c}121.95 \pm 3.76 \\
\mathrm{a}\end{array}$ \\
\hline HDL-Cholesterol (HDL-C) (mg/dl) & $42.3 \pm 0.47$ & $\begin{array}{c}39.4 \pm 0.34 \\
\mathrm{a}\end{array}$ & $\begin{array}{c}39.6 \pm 0.37 \\
\mathrm{a}\end{array}$ & $\begin{array}{c}39.4 \pm 0.33 \\
\mathrm{a}\end{array}$ & $\begin{array}{c}39.6 \pm 0.42 \\
a\end{array}$ \\
\hline
\end{tabular}

Values are presented as mean \pm SE $(n=10)$. The abbreviations denote Glim: rats treated with glimepiride $(0.1 \mathrm{mg} / \mathrm{kg})$, Sita $10:$ rats treated with sitagliptin $(10 \mathrm{mg} / \mathrm{kg})$ and Sita 30: rats treated with sitagliptin (30 mg/kg). a: significantly different from the control group by ANOVA (LSD), p value < 0.05 . 


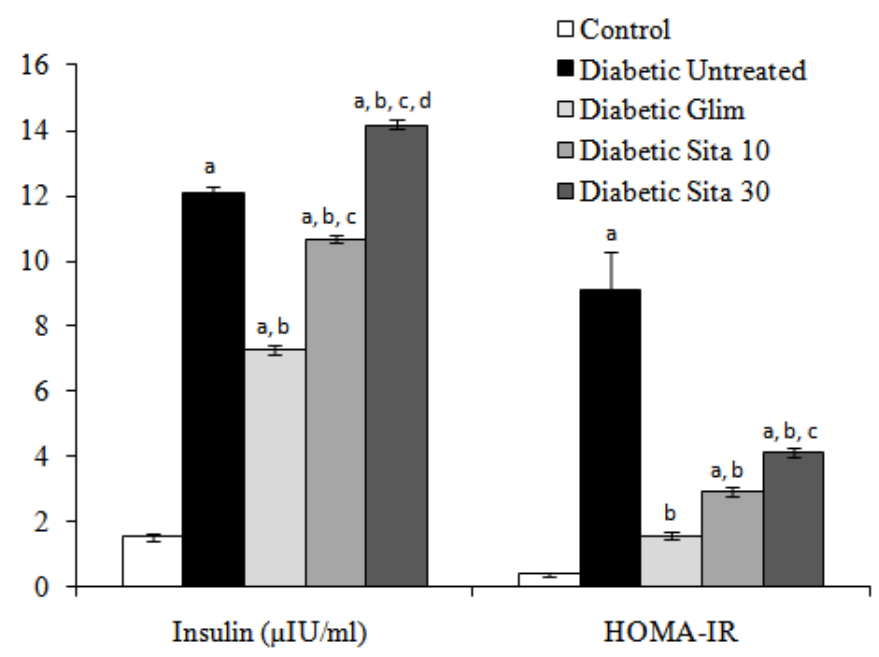

Figure 4. Serum insulin levels $(\mu \mathrm{IU} / \mathrm{ml})$ and HOMA-IR values of different studied groups at the end of the treatment period (4 weeks). Values are presented as mean \pm SE $(n=10)$. The abbreviations denote Glim: rats treated with glimepiride $(0.1 \mathrm{mg} / \mathrm{kg})$, Sita 10 : rats treated with sitagliptin $(10$ $\mathrm{mg} / \mathrm{kg}$ ) and Sita 30: rats treated with sitagliptin (30 mg/kg). a: significantly different from the control group, b: significantly different from the diabetic untreated group, c: significantly different from the Glim group, d: significantly different from the Sita 10 group, using ANOVA (LSD), p value $<0.05$

At the start of the experiment, all diabetic rats showed higher insulin levels compared to the control group (Table 1). By the end of the treatment period, all diabetic groups (untreated and treated) exhibited a significantly higher serum insulin levels compared to the control rats. Diabetic rats treated with glimepiride and sitagliptin at dose of 10 $\mathrm{mg} / \mathrm{kg}$ showed significant lower levels than the untreated diabetic rats, while rats treated with sitagliptin at a dose of $30 \mathrm{mg} / \mathrm{kg}$ showed significant higher level than the untreated diabetic rats (Figure 4).
The insulin resistance index calculated by the HOMA model (HOMA-IR) using the level of fasting insulin $(\mu \mathrm{IU} / \mathrm{ml})$ and glucose level $(\mathrm{mmol} / \mathrm{l})$ indicated that all diabetic groups started the experiment with significantly higher HOMA-IR values compared to the control group (Table 1). At the end of the treatment period, all of the treated rats showed a significant decline in the insulin resistance index compared to the untreated rats with the least value observed in rats treated with glimepiride (Figure 4).

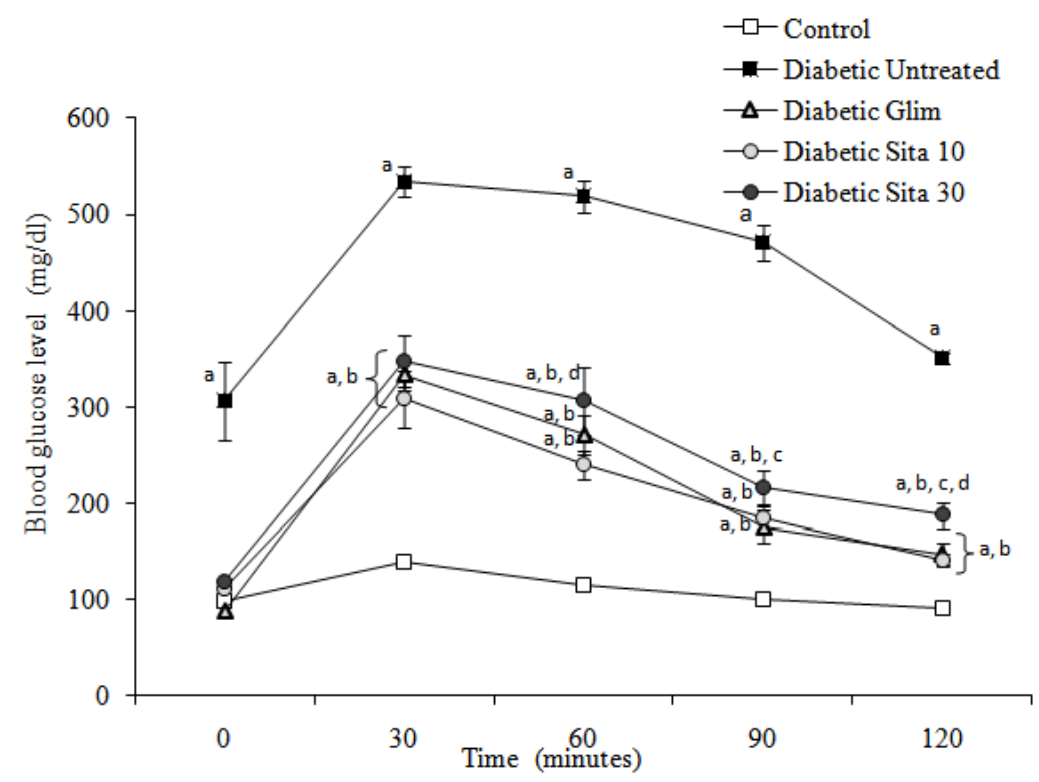

Figure 5. Change in blood glucose level (mg/dl) during the oral glucose tolerance test (OGTT) of different studied groups done at the end of the treatment period (4 weeks). Values are presented as mean \pm SE $(n=10)$. The abbreviations denote Glim: rats treated with glimepiride $(0.1 \mathrm{mg} / \mathrm{kg})$, Sita 10: rats treated with sitagliptin (10 mg/kg) and Sita 30: rats treated with sitagliptin (30 mg/kg). a: significantly different from the control group, b: significantly different from the diabetic untreated group, c: significantly different from the Glim group, d: significantly different from the Sita 10 group, using ANOVA (LSD), p value $<0.05$

From the results of the OGTT constructed at the end of treatment period, it was clear that the untreated diabetic rats was suffering from impaired fasting glucose tolerance and also impaired glucose tolerance during the two hour period of the test (Figure 5), while the treated diabetic rats showed no impairment in the fasting glucose tolerance but showed impaired glucose tolerance after glucose administration which was lesser extent than that observed in the untreated rats (Figure 5). The best response in the OGTT was associated with sitagliptin treatment at a dose 
of $10 \mathrm{mg} / \mathrm{kg}$ followed by glimepiride treatment and the least response was observed with sitagliptin treatment at $30 \mathrm{mg} / \mathrm{kg}$ (Figure 5).

\subsection{Lipid Profile}

At the beginning of the experiment, the baseline values of the lipid profile (triglycerides, total cholesterol, HDL-C and LDL-C) showed significantly higher levels of triglycerides, cholesterol and LDL-C and lower level of HDL-C in the untreated diabetic rats than the control group (Table 1).
At the end of the treatment period, glimepiride significantly corrected the levels of triglyceride, cholesterol and LDL-C, while showed no significant effect on HDL-C (Table 2). Sitagliptin treatment at a dose of 10 $\mathrm{mg} / \mathrm{kg}$ slightly but significantly decreased the level of triglycerides and completely normalized the levels of cholesterol and LDL-C, while the dose of $30 \mathrm{mg} / \mathrm{kg}$ had no effect on triglycerides level and it decreased the cholesterol and LDL-C levels. Sitagliptin at the administered doses showed no effect on the HDL-C level (Table 2).

Table 2. Lipid profile of different studied groups at the end of the treatment period (4 weeks)

\begin{tabular}{|c|c|c|c|c|c|}
\hline \multirow{2}{*}{ Parameter } & \multirow{2}{*}{ Control } & \multicolumn{4}{|c|}{ Diabetic } \\
\hline & & Untreated & Glim & Sita 10 & Sita 30 \\
\hline Triglycerides (TGs) (mg/dl) & $39.9 \pm 0.57$ & $\begin{array}{c}118 \pm 5.2 \\
\mathrm{a}\end{array}$ & $\begin{array}{c}38.4 \pm 3.29 \\
b\end{array}$ & $\begin{array}{c}100.3 \pm 1.69 \\
\text { a, b, c }\end{array}$ & $\begin{array}{c}110.8 \pm 1.08 \\
\text { a, c, d }\end{array}$ \\
\hline Total Cholesterol (mg/dl) & $142.2 \pm 3.76$ & $\begin{array}{c}185.5 \pm 4.07 \\
\mathrm{a}\end{array}$ & $\begin{array}{c}105.2 \pm 3.55 \\
\text { a, b }\end{array}$ & $\begin{array}{c}112.3 \pm 0.94 \\
\text { a, b }\end{array}$ & $\begin{array}{c}122.4 \pm 0.75 \\
\text { a, b, c, d }\end{array}$ \\
\hline LDL-Cholesterol (mg/dl) & $91.81 \pm 3.69$ & $\begin{array}{c}123.1 \pm 4.2 \\
\mathrm{a}\end{array}$ & $\begin{array}{c}59.12 \pm 3.68 \\
\text { a, b }\end{array}$ & $\begin{array}{c}54.69 \pm 1.06 \\
\text { a, b }\end{array}$ & $\begin{array}{c}63.3 \pm 0.71 \\
\text { a, b, d }\end{array}$ \\
\hline HDL-Cholesterol (mg/dl) & $42.5 \pm 0.3$ & $\begin{array}{c}39.1 \pm 0.18 \\
\mathrm{a}\end{array}$ & $\begin{array}{c}38.6 \pm 0.34 \\
\mathrm{a}\end{array}$ & $\begin{array}{c}37.8 \pm 0.13 \\
\text { a, b }\end{array}$ & $\begin{array}{c}37 \pm 0.21 \\
\text { a, b, c }\end{array}$ \\
\hline
\end{tabular}

Values are presented as mean \pm SE $(\mathrm{n}=10)$. The abbreviations denote Glim: rats treated with glimepiride $(0.1 \mathrm{mg} / \mathrm{kg})$, Sita $10: \mathrm{rats}$ treated with sitagliptin (10 mg/kg) and Sita 30: rats treated with sitagliptin (30 mg/kg). a: significantly different from the control group, b: significantly different from the diabetic untreated group, c: significantly different from the Glim group, d: significantly different from the Sita 10 group, using ANOVA (LSD), $\mathrm{p}$ value $<0.05$.

\subsection{Cyclic Adenosine Monophosphate (cAMP) Level}

In the normal control rats, the highest content of cAMP was observed in the BAT (Figure 6). The untreated diabetic rats showed great elevation in cAMP levels of the liver and WAT (12.49 \pm 0.6 and $27.06 \pm 4.3$, respectively) to be 1.3 and 3.9 fold the control values $(9.53 \pm 0.7$ and $6.9 \pm 0.4$, respectively), respectively, while in BAT, the cAMP level (9.38 \pm 0.7$)$ was decreased to be 0.4 fold the control value (21.56 \pm 1.3$)$ (Figure 6).

Glimepiride treated diabetic rats showed significant decreased levels of cAMP in the liver and WAT, to be near the control value in the adipose tissue and even lower than the control value in the liver. The BAT of glimepiride treated rats showed a significantly higher cAMP level than the untreated rats, but lower than the control rats (Figure 6). Sitagliptin treated diabetic rats showed lower hepatic levels of cAMP than the untreated rats and even the control rats at the two doses used. Moreover, the rats showed lower cAMP content in the WAT compared to the untreated rats; the rats treated with sitagliptin at a dose of $30 \mathrm{mg} / \mathrm{kg}$ showed no significant difference from the control rats (Figure 6). Sitagliptin treated rats showed a significant lower level of cAMP in the BAT compared to the untreated and the control rats (Figure 6).

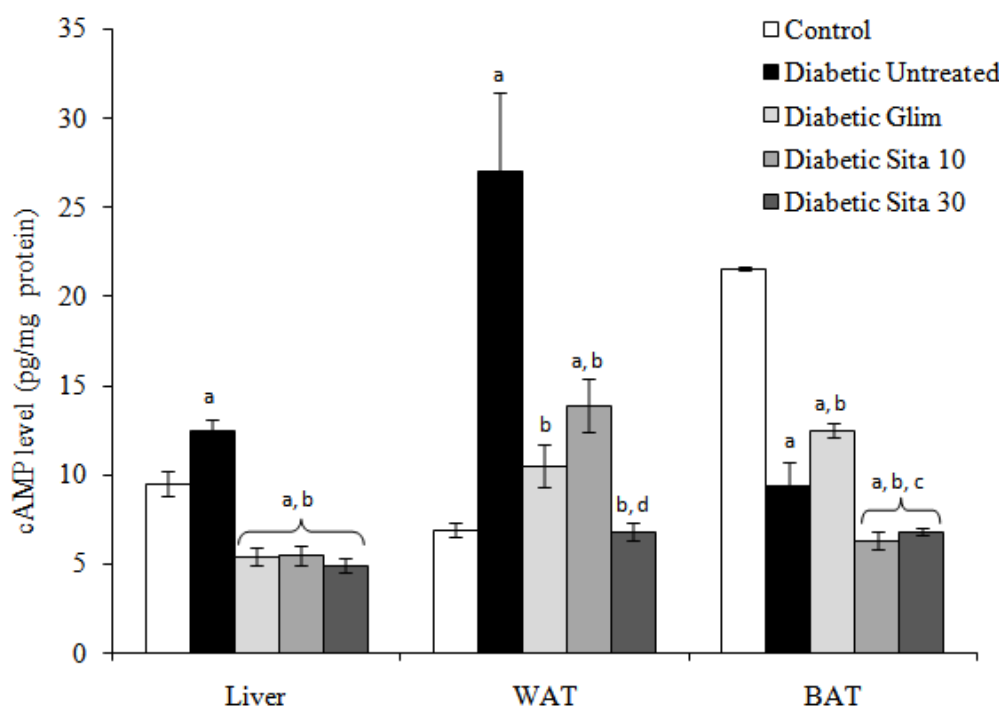

Figure 6. Cyclic adenosine monophosphate (cAMP) levels (pg/mg protein) in the liver, white adipose tissue (WAT) and brown adipose tissue (BAT) of different studied groups. Values are presented as mean \pm SE $(\mathrm{n}=10)$. The abbreviations denote Glim: rats treated with glimepiride $(0.1 \mathrm{mg} / \mathrm{kg})$, Sita 10 : rats treated with sitagliptin (10 mg/kg) and Sita 30: rats treated with sitagliptin (30 mg/kg). a: significantly different from the control group, b: significantly different from the diabetic untreated group, c: significantly different from the Glim group, d: significantly different from the Sita 10 group, using ANOVA (LSD), p value $<0.05$ 


\section{Discussion}

T2DM is a multiorgan disorder which is characterized by early direct or indirect defects in muscles, hepatocytes, adipocytes, and $\beta$-cells [1]. It has been shown that dysregulation in insulin signaling mechanisms results in glucose intolerance, insulin resistance and T2DM [11]. The increasing prevalence of T2DM has stimulated the development of many new approaches to safely treat hyperglycemia. The main goal of these therapies is to reduce glucose levels, and therefore prevent the development of diabetes complications. Several antidiabetic drugs are very well accepted worldwide in terms of low incidence of adverse effects and therapeutic efficiency, and the mechanism of action for most of these drugs has been demonstrated. However, individual responses to these medications can differ significantly, possibly as a result of the heterogeneity of T2DM pathophysiology [12].

This study was designed to demonstrate sitagliptin effects on glucose homeostasis parameters, lipid profile and some insulin signaling components in HFD/STZ diabetic rats, compared to the sulfonylurea glimepiride. Induction of diabetes was performed according to modified Srinivasan, et al. method [9]; HFD was combined with a single low dose of STZ injected intraperitoneally at $45 \mathrm{mg} / \mathrm{kg}$ of body weight. While HFD induces insulin resistance, the low dose of STZ causes mild impairment in $\beta$-cell function [9]. The HFD/STZ diabetic rats exhibited overt hyperglycemia, dyslipidemia, hyperinsulinemia, and insulin resistance (as indicated by increased HOMA). Therefore, this rat model exhibited features of T2DM that would closely reflect the metabolic characteristics of T2DM in humans, and it could be used for pharmacological testing.

The higher fasting insulin level was evident in our model of diabetes, which does not agree with Srinivasan, et al. method [9] in which diabetic rats exhibited nearnormal insulin level after STZ injection. This is could be elucidated as administration of HFD in our model for 4 weeks instead of 2 weeks, exacerbated insulin resistance in diabetic rats even after administration of low dose of STZ. Therefore, insulin is unable to act properly on resistant tissues and this resulted in poor glucose utilization, so $\beta$-cells initially compensated for insulin resistance by increasing insulin secretion. Multiple organs contribute to the development of peripheral insulin resistance, with the major contributors being skeletal muscle, liver, and adipose tissues. Insulin sensitivity is the ability of insulin to lower plasma glucose levels by inhibiting hepatic glucose production and stimulating glucose uptake in skeletal muscle and adipose tissues. On the contrary, insulin resistance describes an impaired biological response to insulin, but there is sufficient variability in normal sensitivity to insulin that there is no specific cut-off at which sensitivity ends and resistance begins [13]. Moreover, there is no absolute definition of hyperinsulinemia, since an insulin concentration that is raised for an individual is usually still within the wide range of normality. While hyperinsulinemia may compensate for resistance to some actions of insulin, it can result in overexpression of actions that retain normal reactivity to insulin [14].
Sitagliptin and glimepiride are insulin secretagogues which enhance insulin secretion from $\beta$-cells by different mechanisms. Sitagliptin inhibits DPP-4 enzyme, thus increasing levels of incretin hormones [7]. Therefore, sitagliptin causes glucose-dependent stimulation of insulin secretion, inhibition of glucagon secretion and preservation of $\beta$-cell mass through stimulation of cell proliferation and inhibition of apoptosis [8]. Glimepiride is a second-generation sulfonylurea acts directly by binding to the ATP-dependent potassium channels $\left(\mathrm{K}^{+}{ }_{\text {ATP }}\right)$ on the $\beta$-cells. The closure of these channels by sulfonylureas results in depolarization of the $\beta$-cells and a successive calcium influx which leads to glucoseindependent insulin release [15]. Moreover, sulfonylureas are reported to inhibit glucagon secretion from pancreatic $\alpha$-cells [16,17].

We found that glimepiride had - to some extent - a better glucose-lowering effect than sitagliptin appeared in weekly determined FBG levels. This finding was in accord with results from previous studies compared sitagliptin with the sulfonylurea glibenclamide in T2DM patients [18]. Both glucose-utilizing effect and insulin secretagogue action of glimepiride and sitagliptin contributed to lower HOMA-insulin resistance value in diabetic rats.

As expected in our model of diabetes (HFD/STZ diabetic model), the disrupted glucose homeostasis is associated with derangements in the lipid profile manifested as increased triglycerides, total cholesterol and LDL-C, as well as decreased HDL-C (collectively called dyslipidemia). These derangements may be a cause or a consequence of the diabetic state and insulin resistance [19]. The diabetic rats treated with glimepiride $(0.1 \mathrm{mg} / \mathrm{kg})$ and sitagliptin (10 mg/kg) showed near-normal plasma lipid profile. It is reported that many T2DM patients continue to have abnormal plasma lipid profiles, although they have achieved their glycemic goals [20]. This pattern is - to some extent - consistent with our findings in rats receiving sitagliptin in which increasing drug dose resulted in an abnormal lipid profile manifested mainly by significant increment of plasma triglycerides level. In line with this, clinical studies of DPP-IV inhibitors have demonstrated modest improvements (decrement in levels of total cholesterol, LDL-C and triglycerides) in lipid panels and not all studies have demonstrated a significant benefit [21].

Besides the classical effects of sitagliptin and glimepiride, they have other ways to ameliorate the diabetic state through their effects on peripheral insulin actions. It was documented that, gluconeogenesis and hepatic glucose production were inhibited by sitagliptin in HFD-induced obese rats due to decreased glycerol availability as a result of reduced glycerol release from adipose tissues [22]. Furthermore, studies suggested an insulin sensitizing action of glimepiride possibly by stimulation of GLUT4 transport protein activation and/or translocation in fat and muscle [23].

cAMP is an important player in the regulation of cellular metabolism and hormonal action in the peripheral tissues. Glucagon acts on the liver and adipose tissues where it binds to its receptors. It activates adenylate cyclase thus elevating intracellular cAMP level. It enhances lipolysis, glycogenolysis as a result of phosphorylase stimulation [24], and gluconeogenesis 
through induction of phosphoenolpyruvate carboxykinase (PEPCK) [3]. On the other hand, it inhibits glucose uptake into adipocytes, thus decreasing triglyceride synthesis [2]. Insulin antagonizes catabolic actions of glucagon - mainly by activation of $\mathrm{PDE} 3 \mathrm{~B}$ via $\mathrm{Akt} / \mathrm{PKB}$, thus reducing cAMP levels [3]. Therefore, hepatic cAMP levels could be used as an indicator of glucagon/insulin ratio and consequently could be used to evaluate the efficiency of antidiabetic drugs.

The results indicated that the diabetic state differentially affects cAMP level according to the type of the tissue. There are two kinds of adipose tissue; WAT which plays a significant role in energy storage in the form of triglycerides, and BAT which plays a substantial role in heat production [5]. Both WAT and BAT are innervated by the sympathetic nervous system [25]. In lipolytically active adipocytes, elevated cAMP levels result in activation/phosphorylation of PKA, which activates hormone sensitive lipase (HSL), and consequently initiate lipolysis. Unrestrained lipolysis, which leads to increase circulating free fatty acids in the absorptive state, has been linked to insulin resistance [26]. In BAT, elevated PKA-activity stimulates expression of UCP1 that uncouples oxidative phosphorylation resulting in heat production [5].

The data of cAMP content in the peripheral tissues clearly indicated higher cAMP level in BAT than in the liver and WAT. It has been demonstrated that sympathetic innervation is more abundant in BAT than in WAT [27]. Moreover, the effect of catecholamines in adipose tissues depends on the type of adrenergic receptor (AR); they have antilipolytic effect in WAT through the $\alpha_{2}$-ARs which inhibit adenylyl cyclase activity [28] whereas, the $\beta_{3}$-ARs are abundantly expressed in BAT which activate adenylate cyclase [29].

The results indicated that, in diabetic rats, while the hepatic and WAT contents of cAMP are greatly elevated, the levels in BAT are depleted. The observed high level of cAMP in the liver and WAT could be explained by insulin resistance state which favors the lipolytic pathway as a result of increasing glucagon/insulin ratio, the produced free fatty acids and glycerol may further exaggerate the insulin resistance in tissues in a vicious cycle. On the other hand, BAT of diabetic rats showed lowered cAMP which could be explained by the fact that BAT are more insulin sensitive than WAT [30] and may retain insulin sensitivity even under insulin resistance. This decline in cAMP in BAT may result in a shift in its metabolic capacity from lipolytic and catabolic into lipogenic and anabolic pathways which could change cells phenotype from brown adipocyte (metabolically active) into white adipocyte (storage) phenotype.

\section{Conclusion}

From the above discussion, we can conclude that the glucose-lowering effects of the two antidiabetic drugs; sitagliptin and glimepiride are - to some extent comparable and are not dependant only on their insulin secretagogue action but also on their effects on peripheral insulin signaling components. They have cAMP-lowering effect in the liver and WAT, which may have protection against vascular complications of diabetes. The mode of action and the molecular targets of sitagliptin in peripheral tissues need to be elucidated. Also, the discrepancy between the low and high doses of sitagliptin needs an explanation.

\section{Competing Interests}

The authors declare that they have no competing interests.

\section{Authors' Contribution}

MIS carried out most experiments and participated in the experimental design. MAK designed the experiments and contributed in writing and revising the manuscript. MYH and MHH performed the statistical analysis of data and helped in writing the manuscript. RRS participated in dose selection, follow up of the animals, data assembly and manuscript revision. All authors have read and approved the final manuscript.

\section{List of Abbreviations}

T2DM, Type 2 diabetes mellitus; HFD, High-fat diet; STZ, Streptozotocin; IRS, Insulin receptor substrate proteins; MAPK, Mitogen activated protein kinase; PKA, Protein kinase A; PKB, Protein kinase B; aPKC, atypical protein kinase C; cAMP, cyclic adenosine monophosphate; BAT, Brown adipose tissue; WAT, White adipose tissue; PDEs, Phosphodiesterases; PDE3B, Phosphodiesterase 3B; DPP-4, Dipeptidyl peptidase-4; GIP, Glucosedependent insulinotropic peptide; GLP-1, Glucagon-like

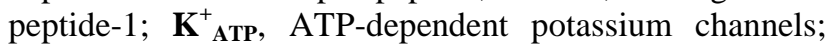
PEPCK, phosphoenolpyruvate carboxykinase; AR, Adrenergic receptor; FBG, fasting blood glucose; DMSO, dimethyl sulfoxide; OGTT, Oral glucose tolerance test; IRI, Insulin resistance index, HOMA-IR, Homeostasis model assessment estimate of insulin resistance; ELISA, Enzyme-linked immunosorbent assay; ANOVA, Analysis of variance; LDL-C, Low density lipoprotein-Cholesterol; HDL-C, High density lipoprotein-Cholesterol.

\section{References}

[1] Lin Y., Sun Z. Current views on type 2 diabetes. J Endocrinol 2010, 204 (1): 1-11.

[2] Brass B.J., Abelev Z., Liao E.P., Poretsky L. Endocrine Pancreas. In Principles of Diabetes Mellitus. $2^{\text {nd }}$ edition. Edited by Poretsky L. New York: Springer; 2010: 37-55.

[3] Wu X., Garvey W.T. Insulin Action. In Textbook of Diabetes. $4^{\text {th }}$ edition. Edited by Holt R.I.G., Cockram C.S., Flyvbjerg A., Goldstein R.J. Singapore: Wiley Blackwell; 2010: 104-125.

[4] Ciaraldi T.P. Cellular Mechanisms of Insulin Action. In Principles of Diabetes Mellitus. $2^{\text {nd }}$ edition. Edited by Poretsky L. New York: Springer; 2010: 75-87.

[5] Gesta S., Tseng Y.H., Kahn C.R. Developmental origin of fat: tracking obesity to its source. Cell 2007, 131: 242-256.

[6] Sell H., Deshaies Y., Richard D. The brown adipocyte: update on its metabolic role. Int J Biochem Cell Biol 2004, 36 (11): 20982104.

[7] Drucker D.J., Nauck M.A. The incretin system: glucagon-like peptide-1 receptor agonists and dipeptidyl peptidase-4 inhibitors in type 2 diabetes. Lancet 2006, 368 (9548): 1696-1705. 
[8] Drucker D.J. The biology of incretin hormones. Cell Metab 2006, 3: 153-165.

[9] Srinivasan K., Viswanad B., Asrat L., Kaul C.L., Ramarao P. Combination of high-fat diet-fed and low-dose streptozotocintreated rat: a model for type 2 diabetes and pharmacological screening. Pharmacol Res 2005, 52: 313-320.

[10] Lowry O.H., Rosebrough N.J., Farr A.L., Randall R.J. Protein measurements with Folin-phenol reagent. J Biol Chem 1951, 193: 265-270.

[11] White M.F. Insulin signaling in health and disease. Science 2003, 302 (5651): 1710-1711.

[12] Kahn S.E., Cooper M.E., Del Prato S. Pathophysiology and treatment of type 2 diabetes: perspectives on the past, present, and future. Lancet 2014, 383 (9922): 1068-1083.

[13] Pirola L., Johnston A.M., Van Obberghen E. Modulation of insulin action. Diabetologia 2004, 47: 170-184.

[14] Reaven G.M. Insulin resistance, the insulin resistance syndrome, and cardiovascular disease. Panminerva Med 2005, 47: 201-210.

[15] Bryan J., Crane A., Vila-Carriles W.H., Babenko AP.., AguilarBryan L. Insulin secretagogues, sulfonylurea receptors and $K$ (ATP) channels. Curr Pharm Des 2005, 11: 2699-2716.

[16] Jackson J.E., Bressler R. Clinical pharmacology of sulphonylurea hypoglycaemic agents: part 2. Drugs 1981, 22: 295-320.

[17] Jackson J.E., Bressler R. Clinical pharmacology of sulphonylurea hypoglycaemic agents: part 1. Drugs 1981, 22: 211-245.

[18] Nathan D.M., Buse J.B., Davidson M.B., Ferrannini E., Holman R.R., Sherwin R., Zinman B.; American Diabetes Association; European Association for Study of Diabetes. Medical management of hyperglycemia in type 2 diabetes: a consensus algorithm for the initiation and adjustment of therapy: a consensus statement of the American Diabetes Association and the European Association for the Study of Diabetes. Diabetes Care 2009, 32: 193-203.

[19] Chahil T.J., Ginsberg H.N. Diabetic dyslipidemia. Endocrinol Metab Clin North Am 2006, 35: 491-510.
[20] Mooradian A.D. Dyslipidemia in type 2 diabetes mellitus. Nat Clin Pract Endocrinol Metab 2009, 5 (3): 150-159.

[21] Addison D., Aguilar D. Diabetes and cardiovascular disease: the potential benefit of incretin-based therapies. Curr Atheroscler Rep 2011, 13 (2): 115-122.

[22] Lu Y.L., Zhou D.Q., Zhai H.L., Wu H., Guo Z.K. Decreased hepatic glucose production in obese rats by dipeptidyl peptidaseIV inhibitor sitagliptin. Chin Med J 2012, 125 (10): 1690-1694.

[23] Müller G., Wied S., Wetekam E.M., Crecelius A., Unkelbach A., Pünter J. Stimulation of glucose utilization in 3 T3 adipocytes and rat diaphragm in vitro by the sulphonylureas, glimepiride and glibenclamide, is correlated with modulations of the cAMP regulatory cascade. Biochem Pharmacol 1994, 30 (48): 985-996.

[24] Magnusson I., Rothman D., Gerard D., Katz L., Shulman G. Contribution of hepatic glycogenolysis to glucose production in humans in response to a physiological increase in plasma glucagon concentration. Diabetes 1995, 44: 185-189.

[25] Ballard K., Malmfors T., Rosell S. Adrenergic innervation and vascular patterns in canine adipose tissue. Microvasc Res 1974, 8: 164-171.

[26] Boden G. Fatty acid-induced inflammation and insulin resistance in skeletal muscle and liver. Curr Diab Rep 2006, 6: 177-181.

[27] Hausberg M., Morgan D.A., Mitchell J.L., Sivitz W.I., Mark A.L., Haynes W.G. Leptin potentiates thermogenic sympathetic responses to hypothermia: a receptor-mediated effect. Diabetes 2002, 51: 2434-2440.

[28] Lafontan M., Berlan M. Fat cell adrenergic receptors and the control of white and brown fat cell function. J Lipid Res 1993, 34: 1057-1091.

[29] Sell H., Deshaies Y., Richard D. The brown adipocyte: update on its metabolic role. Int J Biochem Cell Biol 2004, 36: 2098-104.

[30] Orava J., Nuutila P., Lidell M.E., Oikonen V., Noponen T., Viljanen T., Scheinin M., Taittonen M., Niemi T., Enerback S., Virtanen K.A. Different metabolic responses of human brown adipose tissue to activation by cold and insulin. Cell metabolism 2011, 14 (2): 272-279. 